\title{
A Communication Scheduling Scheme for UHV Transmission Line Construction and Security Mechanism Research
}

\author{
Shuhao Zhang ${ }^{1,2, a}$, Gangjun Gong ${ }^{3, b}$, Xiangping $\mathrm{Ni}^{2, \mathrm{c}}$ and Yadi Zhang ${ }^{2, \mathrm{~d}}$ \\ ${ }^{1}$ Wuhan University of Technology, 122 Luoshi Road, Wuhan, Hubei, China 430070 \\ ${ }^{2}$ State Grid AC Engineering Construction Company, Beijing, China 100052 \\ ${ }^{3}$ School of Electronic and Electric Engineering, North China Electric Power Univ., Beijing, China \\ 102206 \\ ashuhao-zhang@sgcc.com.cn, bgonggangjun@126.com, xiangping-ni@sgcc.com.cn, \\ yadi-zhang@sgcc.com.cn
}

Keywords: UHV transmission line, Chain-relay network, Broadband wireless, Security mechanism, System encryption

\begin{abstract}
The constructions of the UHV transmission line are mostly located in remote place without public network coverage, and there are few effective com-munication means. This paper proposes a new scheme which adopts wireless Mesh Wi-Fi network and Digital Microwave Communication Technology based on COFDM, and analyzes their technical characteristics, networking modes and application respectively. Furthermore, this paper presents a merged scheme and the security mechanism which can provide the voice, data and video service for the constructions of the UHV transmission line.
\end{abstract}

\section{Introduction}

The State Grid Corporation of China is planning to build the UHV backbone grid called "three horizontal three longitudinal and two centers", which connects the large-scale energy bases and load centers, to achieve the purposes of the power from west to east and the power from north to east. While the construction of substations, the assembling of double-circuit steel pipe towers and stringing are long period, complex process and difficult construction, which adopt lots of new equipments for the first time. Comparing with old equipments, the size is larger, the weight is heavier and the process is more complex. And the construction of UHV transmission line has many characteristics, such as long distance, big geographical span and adverse working circumstance which are via complex environment, such as jungle, river, lake and so on. So to provide an effective communication manner for production scheduling is difficult.

Because the size and the weight of equipment and construction materials of $1000 \mathrm{KV}$ and $800 \mathrm{KV}$ UHV transmission lines are greater than those in previous projects, the Reference Literature [1] recommends a new two-rope circulated cableway technique. The Reference Literature [2] presents a novel high voltage transmission line monitoring system, which consists of wireless monitoring nodes and optical nodes mounted on the top of transmission towers and utilizes one or two idle fibers in the special fiber cable for transmission lines, to realize on-line supervision of the whole transmission line in complex environments. In Reference Literature [3], the environment-friendly flying machine is used for the HV transmission line stringing. The reference literature [4] proposes a video transmission system based on COFDM for UHV transmission line construction.

This paper analyses the present situation of the UHV transmission line project and the requirement of field communication and puts forward a field communication scheme, which combines the wireless Mesh Wi-Fi chain-relay network with digital microwave communication technology based on COFDM. Furthermore, this paper discusses the application mode and the security mechanism for the scheme which can provide a high quality, reliable communication manner for UHV transmission line production scheduling. 
The construction site of UHV transmission line is temporary and complex, which means that laying cable optical fibers is not practical. So the wireless communication is the preferred way for the nomadic communication network, which must provide a high quality, real-time and reliable communication manner and can communicate with anyone at any time in the construction site.

At present, the broadband wireless communication technology used for the construction site includes satellite communication technology, digital microwave communication technology, 3G public network and wireless Mesh Wi-Fi technology.

1. Satellite communication technology. There are many advantages, such as large capacity, long distance and not subject to geographical constraints. While the communication tariff is more expensive than the cable communication and microwave communication and the price of the equipment is dozens of times that of the other equipment.

2.3G public network. The main feature of the WCDMA and CDMA technology which are mature 3G communication standard is broadband. In the area without signal coverage, 3G signal is poor which cannot satisfy video service in the construction site.

3. Digital microwave communication technology based on COFDM. COFDM is also called coded orthogonal frequency division multiplexing which is the most potential modulation technique. It has good immunity to interference and breaks through in line-of- sight restriction.

4. The wireless Mesh Wi-Fi technology. Mesh network technology is IP-oriented. Wi-Fi is suitable for regional coverage and is a high-speed broadband wireless access.

An effective communication is necessary for construction site in remote area, which can avoid human error and is helpful for stereotactic scheduling.

\section{UHV transmission line communication system design}

Communication Requirements. There are four communication scenes in construction site: The UHV transmission line communication framework is shown in Fig.1.

1. Synchronization scheduling of adjacent points for material allocation and transportation.

2. Communication between the staff on the tower and staff under the tower.

3. Synchronization scheduling of chain points for stringing.

4. The scheduling between dynamic parachute and staff on the tower for stringing.

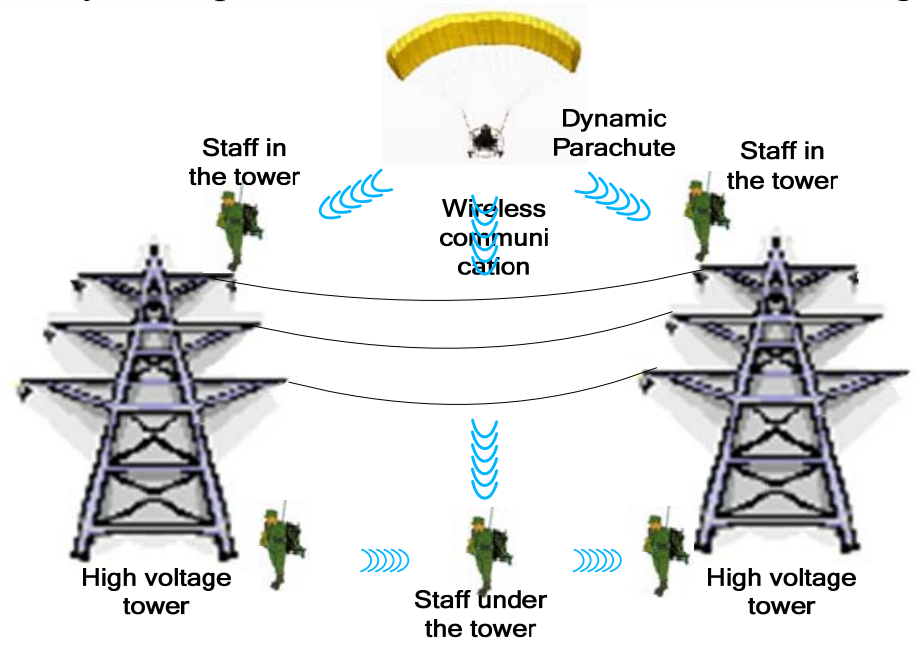

Fig.1 UHV transmission line communication framework

Wireless Mesh Wi-Fi Chain-relay Network. Wireless Mesh Networks (WMNs) are dynamically self-organized and self-configured broadband wireless network, which is IP-oriented and consist of mesh routers and mesh client. This feature brings many advantages to WMNs, such as low up-front cost, easy network maintenance, robustness, reliable, service coverage, etc. While the transmission rate is drop sharply after several relay transmissions. Generally, the number of times relays can forward a packet is no more than four times. The transmission rate after several times relay transmission is shown in Fig.2. 




Fig. 2 The transmission rate after several times relay transmission

Wi-Fi is a broadband wireless access technology. It has many advantages, such as high speed, low power consumption, no wiring, etc, while the coverage area is small and the network is vulnerable to attack. So we take the Wi-Fi as access point and mesh network as relay connecting the Wi-Fi access point, which can reach larger coverage area. The Wireless Mesh Wi-Fi chain-relay network architecture is shown in Fig.3.

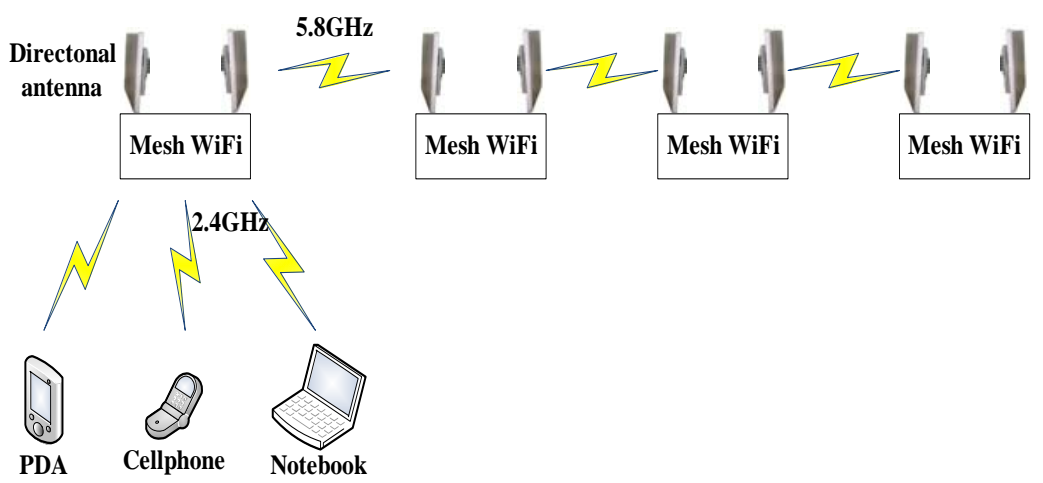

Fig.3 The Wireless Mesh Wi-Fi chain-relay network architecture

Long Distance Broadband Wireless Communication Scheme Based on COFDM. COFDM is the abbreviation for coded orthogonal frequency division multiplexing and can work in multiple frequency bands $(200 \mathrm{MHz}, 300 \mathrm{MHz}, 400 \mathrm{MHz}, 800 \mathrm{MHz}$, etc) and at several transmission rates (16Mbit/s, 8Mbit/s, 4Mbit/s, 2Mbit/s, etc). COFDM with networking capacity and diffraction capability is available in jungle, mountains and the COFDM relay equipment can achieve larger coverage area and longer transmission distance. The COFDM communication system is shown in Fig.4.



Fig. 4 The Long distance COFDM communication system

Characteristics of Mesh Wi-Fi and COFDM. There are benefits and drawbacks to Mesh Wi-Fi 
access technology and COFDM, specifically as shown in Table.1 \&2.

\begin{tabular}{lllll}
\hline Title & Frequency Band & Transfer rate & $\begin{array}{l}\text { Transmission } \\
\text { distance }\end{array}$ & \\
\hline COFDM & $200 \mathrm{MHZ} \sim 800 \mathrm{MHz}$ & $\leq 16 \mathrm{Mbps}$ & $\leq 20 \mathrm{~km}$ & $<5 \%$ \\
Mesh & $2.4 \mathrm{GHz}, 5.8 \mathrm{GHz}$ & $\leq 100 \mathrm{bps}$ & $\leq 4 \mathrm{~km}$ & $>20 \%$ \\
Wi-Fi & & & & \\
\hline \multicolumn{1}{c}{ Table.1 } & Comparing Mesh Wi-Fi access technology with COFDM \\
\hline Title & Networking & Security & $\begin{array}{l}\text { Diffraction } \\
\text { capability }\end{array}$ & Cost \\
\hline COFDM & P2P or PMP & $\begin{array}{l}\text { support } \\
\text { encryption }\end{array}$ & strong & low \\
Mesh & IP Net-working & $\begin{array}{l}\text { support } \\
\text { encryption }\end{array}$ & weak & low \\
Wi-Fi & & & \\
\hline
\end{tabular}

Table.2 Comparing Mesh Wi-Fi access technology with COFDM

Integration solution. Because of the technical features of COFDM and Mesh Wi-Fi, the Integration solution which adopts COFDM and Mesh Wi-Fi wireless broadband network access can provide bidirectional data, audio and high-definition video services anytime in construction site. The specific design is that the return journey of broadband wireless local access network adopts COFDM and Mesh Wi-Fi is used in the on-site scheduling system. The architecture of the integration system is shown in Fig.5.

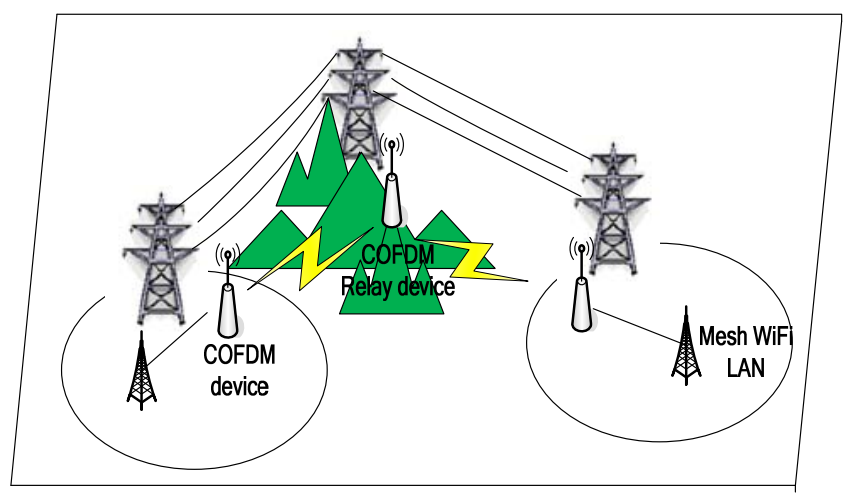

Fig.5 The merged communication network based on COFDM and Mesh Wi-Fi

In the progress of on-site scheduling, the system adopts COFDM technology to realize point-to-point wireless communication and on-site scheduling return-journey network, which transmits on-site information to command center. The Wi-Fi broadband wireless network can provide bidirectional data flow of on-site command and a large-scale signal coverage area, which cover the construction site and extend production scheduling to anywhere in construction site.

So the integration scheme can ensure the accuracy of production scheduling and the security of power construction.

\section{The analysis of security mechanism of UHV transmission line communication system}

Wi-Fi is a wireless network technology based on IEEE802.11 standard and wire-less Mesh network is a further extension of Wi-Fi, working at $2.4 / 5.8 \mathrm{GHz}$ ISM open band which can be used by any person or organization in the signal coverage area, so the security problems should be paid great attention to. The development of Wi-Fi Mesh security mechanism is shown in Fig.6. There are some common security problems, such as DOS attack, key cracking, lack of key management, attack-in-middle attack, etc. The common solutions include SSID, WPA, IP-MAC binding, MAC filtering, internal encryption in key devices, etc, which protect the security of communication link. 




Fig.6 The development course of security measures

DVB-T based on COFDM adopts digital TV encryption to ensure the transmission security, which will not cause signal loss in theory. Therefore, in construction site of UHV transmission line, digital TV encryption can be used to ensure the security of on-site scheduling return-journey network. The Wi-Fi broadband wireless network can adopt end-to-end IP-MAC bind, WPA2 AES block cipher and upgrading Wi-Fi devices for encryption. The overall encryption scheme is shown in Fig.7

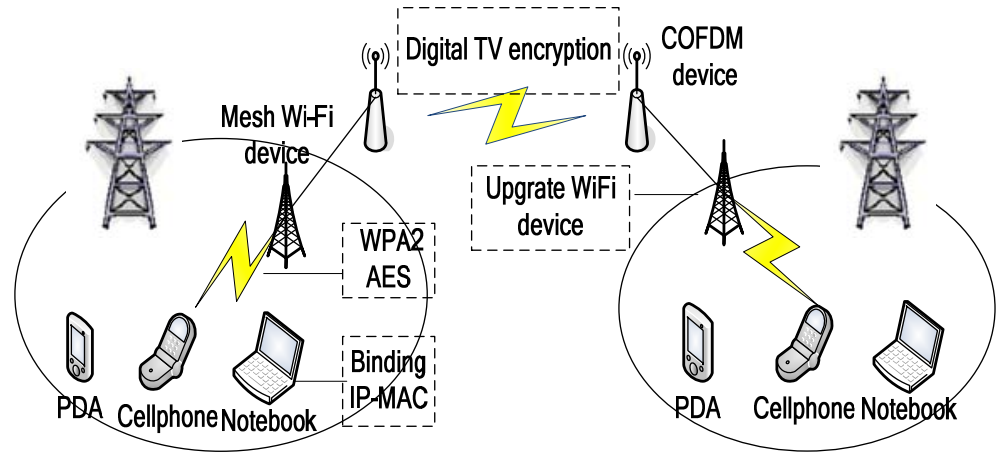

Fig.7 The encryption scheme in UHV transmission system

\section{Conclusion}

This paper analyzed the requirements and the present situation of the communication service in UHV transmission line construction site and presented a solution which combined wireless Mesh Wi-Fi and digital microwave communication technology based on COFDM. This scheme has many advantages, such as low cost, simple networking, wide bandwidth and strong anti-interference ability, which can meet the communication requirements in the area without public network. We also proposed an integration application and analyzed the security mechanism of dispatching scheme, which can ensure the security of the merged scheme.

\section{References}

[1] Fenggang Guo: Power System and Clean Energy Vol. 26 (2010). (In Chinese)

[2] Wang Xuan, Li Hong, Cong Lin: Shandong Electric Power Vol.1 (2010). (In Chinese)

[3] Congjun Hou: Power System and Clean Energy Vol. 27 (2011). (In Chinese)

[4] Xiangping Ni, Yadi Zhang: Modern Power (supplement) Vol. 29 (2012). (In Chinese)

[5] Jie Liu: Television Engineering Vol. 3 (2002). (In Chinese) 